\title{
Species richness, distribution and genetic diversity of Caenorhabditis nematodes in a remote tropical rainforest
}

Marie-Anne Félix ${ }^{1 \dagger}$, Richard Jovelin ${ }^{2 \dagger}$, Céline Ferrari ${ }^{3,4,5}$, Shery Han², Young Ran Cho ${ }^{2}$, Erik C Andersen ${ }^{6}$, Asher D Cutter ${ }^{2}$ and Christian Braendle $3,4,5^{*}$

\begin{abstract}
Background: In stark contrast to the wealth of detail about C. elegans developmental biology and molecular genetics, biologists lack basic data for understanding the abundance and distribution of Caenorhabditis species in natural areas that are unperturbed by human influence.

Methods: Here we report the analysis of dense sampling from a small, remote site in the Amazonian rain forest of the Nouragues Natural Reserve in French Guiana.

Results: Sampling of rotting fruits and flowers revealed proliferating populations of Caenorhabditis, with up to three different species co-occurring within a single substrate sample, indicating remarkable overlap of local microhabitats. We isolated six species, representing the highest local species richness for Caenorhabditis encountered to date, including both tropically cosmopolitan and geographically restricted species not previously isolated elsewhere. We also documented the structure of within-species molecular diversity at multiple spatial scales, focusing on $57 \mathrm{C}$. briggsae isolates from French Guiana. Two distinct genetic subgroups co-occur even within a single fruit. However, the structure of C. briggsae population genetic diversity in French Guiana does not result from strong local patterning but instead presents a microcosm of global patterns of differentiation. We further integrate our observations with new data from nearly 50 additional recently collected C. briggsae isolates from both tropical and temperate regions of the world to re-evaluate local and global patterns of intraspecific diversity, providing the most comprehensive analysis to date for C. briggsae population structure across multiple spatial scales.

Conclusions: The abundance and species richness of Caenorhabditis nematodes is high in a Neotropical rainforest habitat that is subject to minimal human interference. Microhabitat preferences overlap for different local species, although global distributions include both cosmopolitan and geographically restricted groups. Local samples for the cosmopolitan C. briggsae mirror its pan-tropical patterns of intraspecific polymorphism. It remains an important challenge to decipher what drives Caenorhabditis distributions and diversity within and between species.
\end{abstract}

Keywords: Caenorhabditis, Species richness, Population structure, C. briggsae, Nucleotide diversity

\footnotetext{
* Correspondence: braendle@unice.fr

${ }^{\dagger}$ Equal contributors

${ }^{3}$ Institut de Biologie Valrose, CNRS, UMR7277, Parc Valrose, Nice cedex 02 06108, France

Full list of author information is available at the end of the article
}

\section{Biomed Central}




\section{Background}

Understanding the patterns and drivers that govern the abundance and diversity of organisms motivates the study of ecology and evolution. Historically, however, such study has focused primarily on non-model organisms, with some notable exceptions (e.g. Drosophila melanogaster in evolutionary biology). Ecological and evolutionary investigations have particularly neglected the nematode Caenorhabditis elegans and its close relatives until recently [1,2]. And yet, the deep mechanistic knowledge about C. elegans development and life history, coupled with its experimental advantages in the laboratory, make it particularly suited for a more complete integration of molecular genetic approaches with evolutionary and ecological questions. This merging of disciplines is motivated further by interesting phylogenetically variable characteristics of the genus, such as the independent origins of androdioecy (i.e. self-fertilizing reproduction by hermaphrodites and facultative outcrossing with males) in at least three lineages from the more prevalent gonochoristic reproductive mode among other Caenorhabditis species, which reproduce through obligatory mating of females and males $[3,4]$. A major impediment to fully realizing the potential of integrating these fields, however, is the lack of basic information about species distribution and richness in nature.

Studies of Caenorhabditis in nature are difficult, not only because of their small size (ca. $1 \mathrm{~mm}$ length), but also because we know little about the habitats in which they can be found reliably [2]. For this reason, the first population genetic analyses on $C$. elegans were conducted from opportunistic collection or by sampling populations in artificial compost heaps [5-10]. Subsequently, C. elegans was isolated from rotting fruits, as well as in phoretic association with invertebrates such as snails, slugs and isopods $[3,5,11,12]$. Compost heap populations are mostly composed of animals in dauer diapause, but $C$. elegans and $C$. briggsae were recently shown to proliferate and feed in rotting fruits and stems in mainland France [13]. Those rotting fruits were collected primarily in human-built orchards; although rotting stems are found in natural preserves, primary forests no longer exist in Europe [13]. Like many other model organisms (Drosophila melanogaster, Saccharomyces cerevisiae, Mus musculus), C. elegans is thus at least partly human-associated, which likely exerts a strong influence on its range, migration patterns, and population structure $[5,14,15]$. However, we still have very limited information on the distribution of C. elegans and other Caenorhabditis species relating to habitat type and geographic location. Most importantly, we lack information on Caenorhabditis species diversity and population structure from unperturbed habitats.

Here, we report the results of a systematic sampling of Caenorhabditis across spatial scales, from within a single fruit to sampling at metre, kilometre, regional and global scales. In order to minimize the impact of humans and to contrast previously studied temperate sites with a tropical location, we sampled Caenorhabditis populations in a primary Neotropical rain forest in French Guiana, at the Nouragues National Reserve. We quantified the structure of diversity at different genetic and spatial scales, including the species richness of Caenorhabditis at the Nouragues location. In addition, we examined levels of intraspecific molecular polymorphism of $C$. briggsae from French Guiana and from additional recent local sampling of $C$. briggsae from different regions of the world.

\section{Results}

\section{Species diversity in French Guiana}

We assessed a total of 184 samples for nematodes of the genus Caenorhabditis from a broad spectrum of substrate types in French Guiana (Figures 1 and 2, Table 1, Additional files 1 and 2). We isolated five different Caenorhabditis species, in addition to a sixth species $(C$. sp. 12) found in May 2008 that was not found in 2009. Three of these species (C. sp. 12, C. sp. 17, and C. sp. 18) have so far only been reported from French Guiana [3]; all three are gonochoristic. C. sp. 12 belongs to the Drosophilae supergroup of Caenorhabditis species, and is presently the sister species of $C$. angaria, with which it can form F1 hybrids [3]. C. sp. 17 and C. sp. 18 are closely related to each other within the Japonica group of Caenorhabditis (Figure 2). The other three species found in Nouragues are species known from tropical regions on other continents [3], namely, the gonochoristic C. brenneri and the two selfing androdioecious species, $C$. briggsae and $C$. sp. 11. The most commonly encountered species at the Nouragues Natural Reserve were the androdieocious species (C. briggsae, C. sp. 11) and the new gonochoristic species C. sp. 17.

\section{Substrate type and presence of Caenorhabditis}

We found Caenorhabditis nematodes in a wide variety of rotting vegetal substrates (fruits, flowers, plant stems); however, we could not detect any obvious substrate specificity of the different species (Additional file 2). Samples examined immediately after isolation indicated that Caenorhabditis population size in a given sample varied greatly, from one to approximately one thousand individuals, including both dauer stages and proliferating populations with non-dauer juvenile and adult stages (Additional file 2). Of the 130 Nouragues samples, 33 (25\%) contained Caenorhabditis, including 16 of 48 rotting fruits and 11 of 19 rotting flowers. Proliferating populations of Caenorhabditis were found both in rotting fruits and flowers, as well as in one of three samples of erect live flowers (Heliconia sp.). Caenorhabditis 


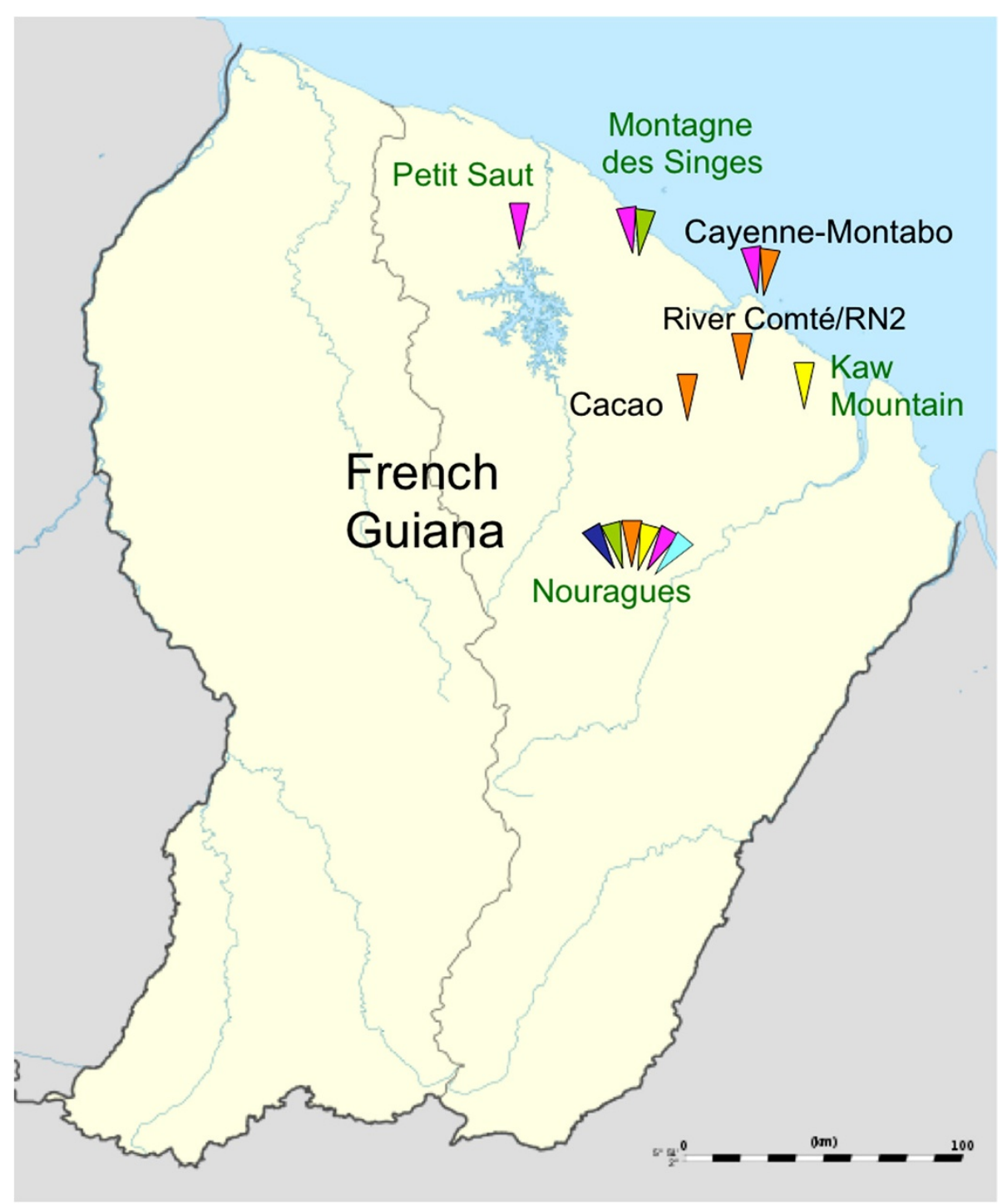

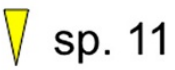

$\nabla$ sp. 17

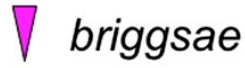

sp. 18

\section{brenneri}

sp. 12

Figure 1 Sampling of Caenorhabditis species in French Guiana. The triangles indicate the presence of different Caenorhabditis species in the sampled areas, colour-coded as indicated in the legend. The names of locations with strong human activity (e.g. towns and villages) are written in black, and other locations (e.g. forests) in green.

nematodes were rarely found in humus or leaf litter (3 of 24) or in association with live or dead insects (0 of 20). In 8 of 33 positive samples, we found two or three different Caenorhabditis species co-occurring in rotting fruits or flowers sampled at the same site (e.g. C. briggsae, spp. 11 and 17, isolated from Cecropia fruits, sample D25, Additional file 2). Many samples also contained nematode species other than Caenorhabditis, including members of the Rhabditina (e.g. Oscheius spp.), Diplogastrina or Aphelenchida.

\section{Distribution of Caenorhabditis species at different} spatial scales

At the kilometre scale, the spatial distribution of Caenorhabditis species appears non-uniform across the landscape in the Nouragues Natural Reserve (Figure 3), 


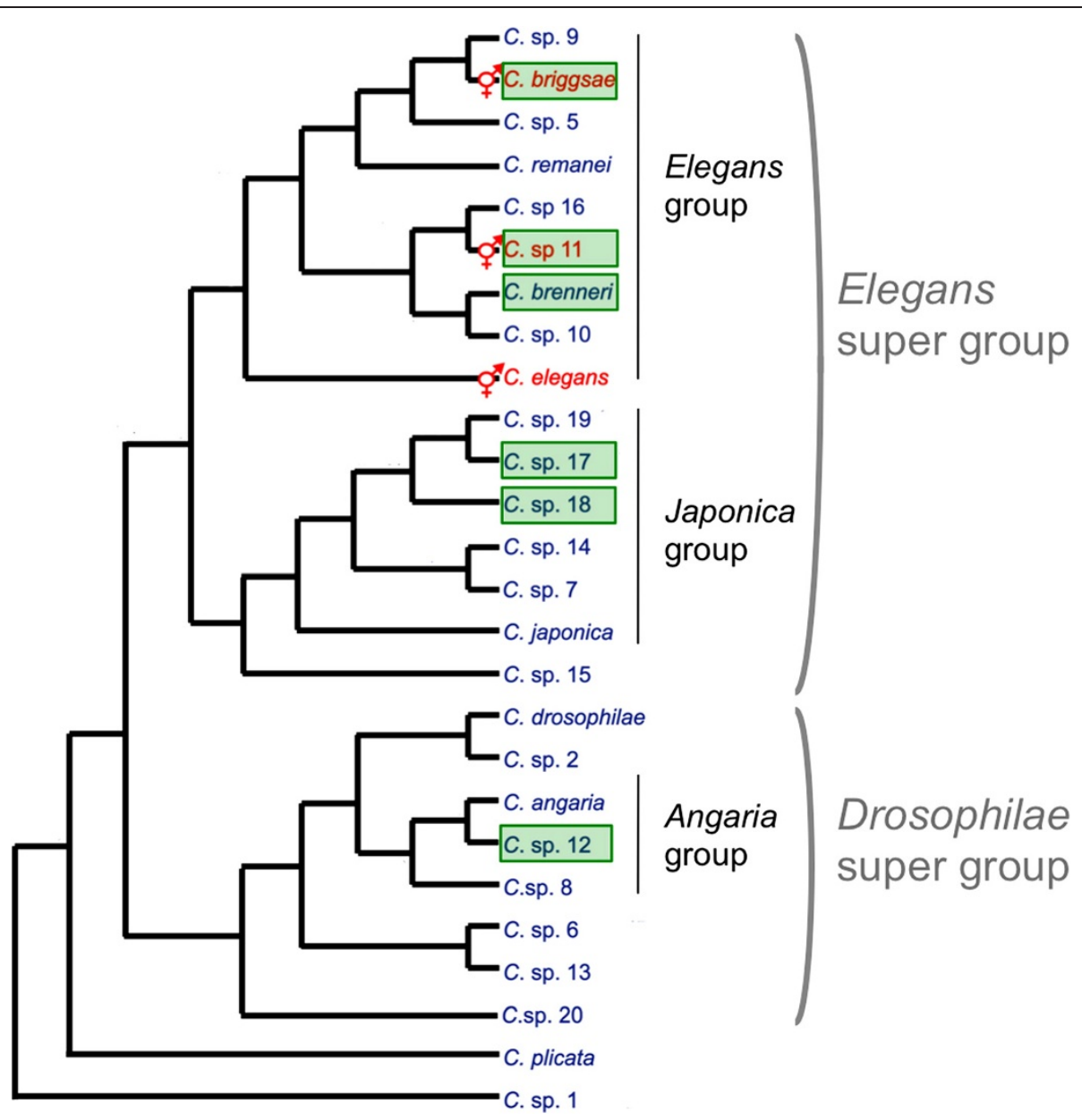

Figure 2 Phylogeny of the Caenorhabditis genus, adapted from Kiontke et al. 2011 [3]. A green box highlights the species found in French Guiana. The selfing species are indicated in red.

suggesting that geographic and ecological factors structure species distributions at this medium scale. Indicative of potential species structuring are the following observations: 1) all seven of the C. briggsae isolations were obtained from the Grand Plateau area, 2) the 11 samples that contained any Caenorhabditis from the
Nouragues river area (a swamp wood called "pinotière", i. e. dominated by Euterpe oleracea palms) included $C$. sp. 11 (and in one instance also $C$. sp. 17), suggesting that $C$. sp. 11 is more common than other Caenorhabditis species in this habitat, and 3) C. sp. 17 was found commonly over the entire sampling area, except perhaps in the river area.

Table 1 Summary of Caenorhabditis species and isolates isolated from different localities in French Guiana

\begin{tabular}{llcll}
\hline Species & Mode of reproduction* & Number of isolates & $\begin{array}{l}\text { Locations in French Guiana } \\
\text { (No. of isolates) }\end{array}$ & Known distribution \\
\hline C. briggsae & androdioecy & 62 & $\begin{array}{l}\text { Nouragues (54), Montagne des Singes (6), Montabo (1), } \\
\text { Petit Saut (1) }\end{array}$ & Cosmopolitan \\
C. sp. 11 & androdioecy & 31 & Nouragues (30), Kaw mountain (1) & Cosmopolitan, tropical \\
C. brenneri & gonochorism & 13 & Nouragues (9), Montabo (1), Comté riverbank (1), Cacao (2) & Cosmopolitan, tropical \\
C. sp. 17 & gonochorism & 30 & Nouragues (28), Montagne des Singes (2) & French Guiana \\
C. sp. 18 & gonochorism & 13 & Nouragues (13) & Nouragues \\
C. sp. 12 & gonochorism & 2 & Nouragues (2) & Nouragues \\
\hline
\end{tabular}

*androdioecy = self-fertile hermaphrodites and males, gonochorism $=$ females and males. 

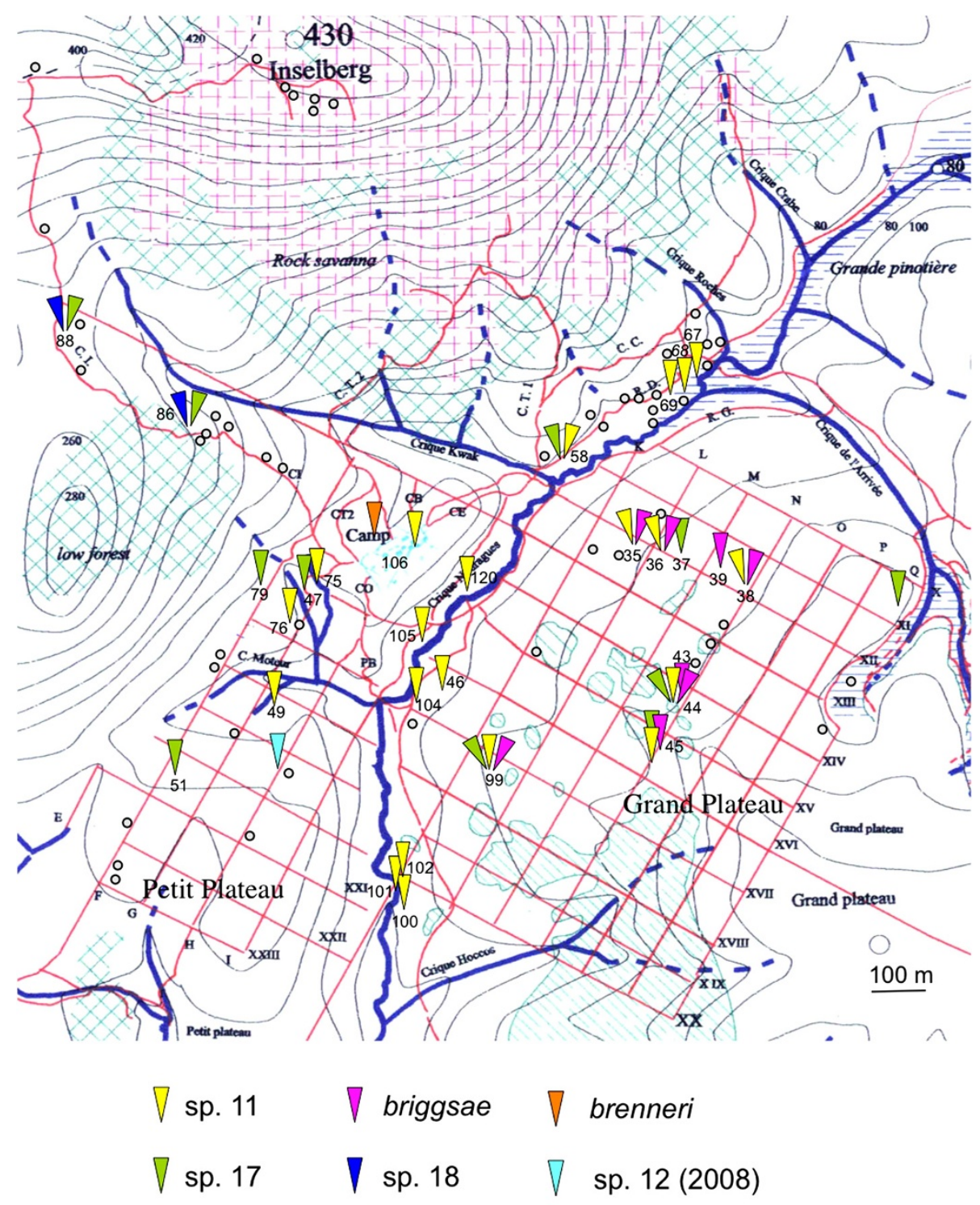

\section{Negative sample}

Figure 3 Spatial distribution of Caenorhabditis species in the Nouragues Natural Reserve. Triangles indicate the presence of a Caenorhabditis species, colour-coded as indicated in the legend. Numbers next to the triangles correspond to the GPS coordinate identifier (see Additional file 2). Small grey dots indicate spots containing only negative samples, i.e. samples lacking Caenorhabditis.

To test for small-scale (i.e. geographically localised) spatial structuring of species and genotype diversity in the Nouragues Natural Reserve, we focused on two sampling sites (H3 and H6) that contained large numbers of rotting Cecropia fruits on the ground (Additional file 2, Figure 4). At site H3, we processed 52 samples, of which 23 contained C. briggsae (7 of 11 fruit samples, 1 of 3 leaf litter samples, 2 of 4 invertebrate samples) and one contained C. sp. 11 (a fruit sample without C. briggsae). At site H6, we processed 47 samples, of which 15 contained C. briggsae (6 of 11 fruit samples, 1 of 3 leaf litter samples, 1 of 3 humus samples) and 4 contained C. sp.
11 (one fruit sample without C. briggsae; co-occurring with $C$. briggsae in 3 fruit samples). At both sites, many substrate samples (60 of 99) also contained other nematode species, including Oscheius spp., which co-occurred with the Caenorhabditis species in 16 of 38 cases.

\section{Pattern of C. briggsae genetic diversity at different spatial scales}

To test for local population structure within a species, we focused on the C. briggsae isolates resulting from the above small-scale spatial analysis, then considered these together with isolates from the entire Nouragues area 


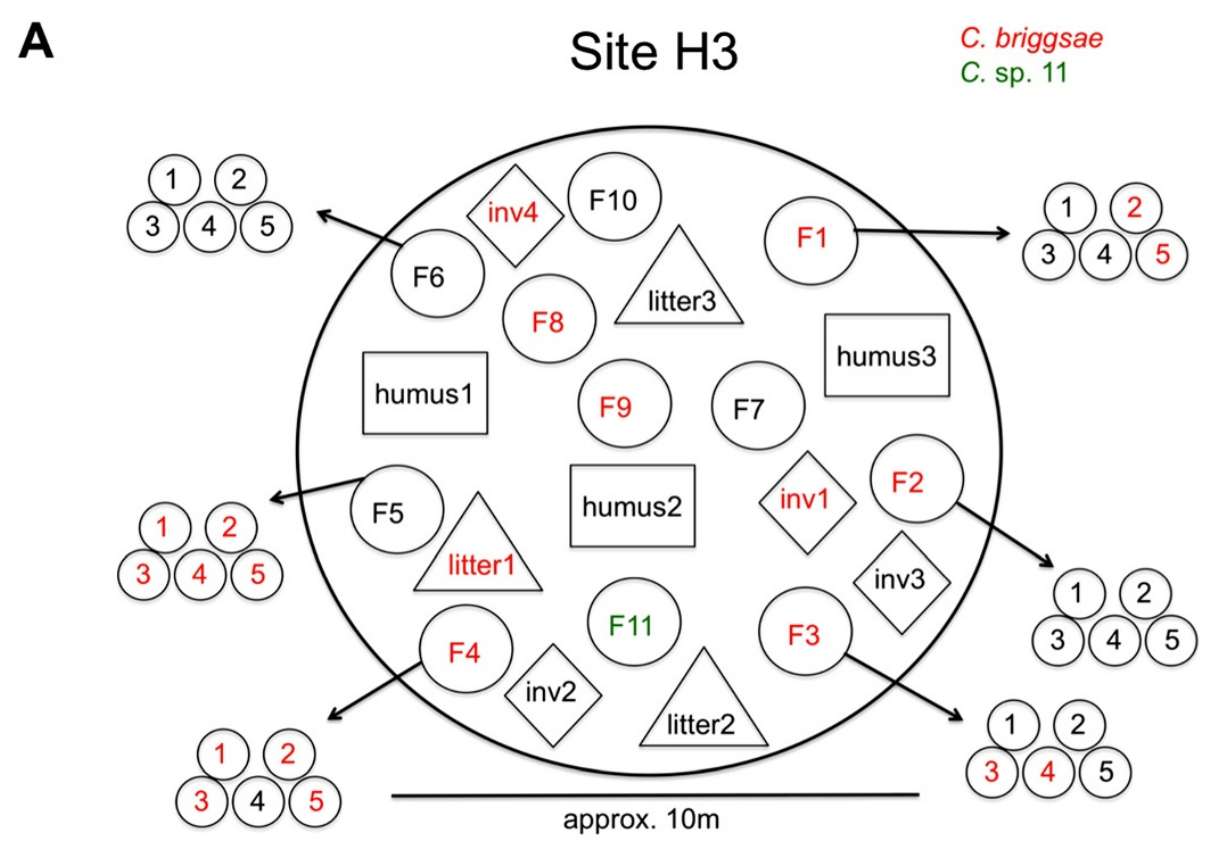

B

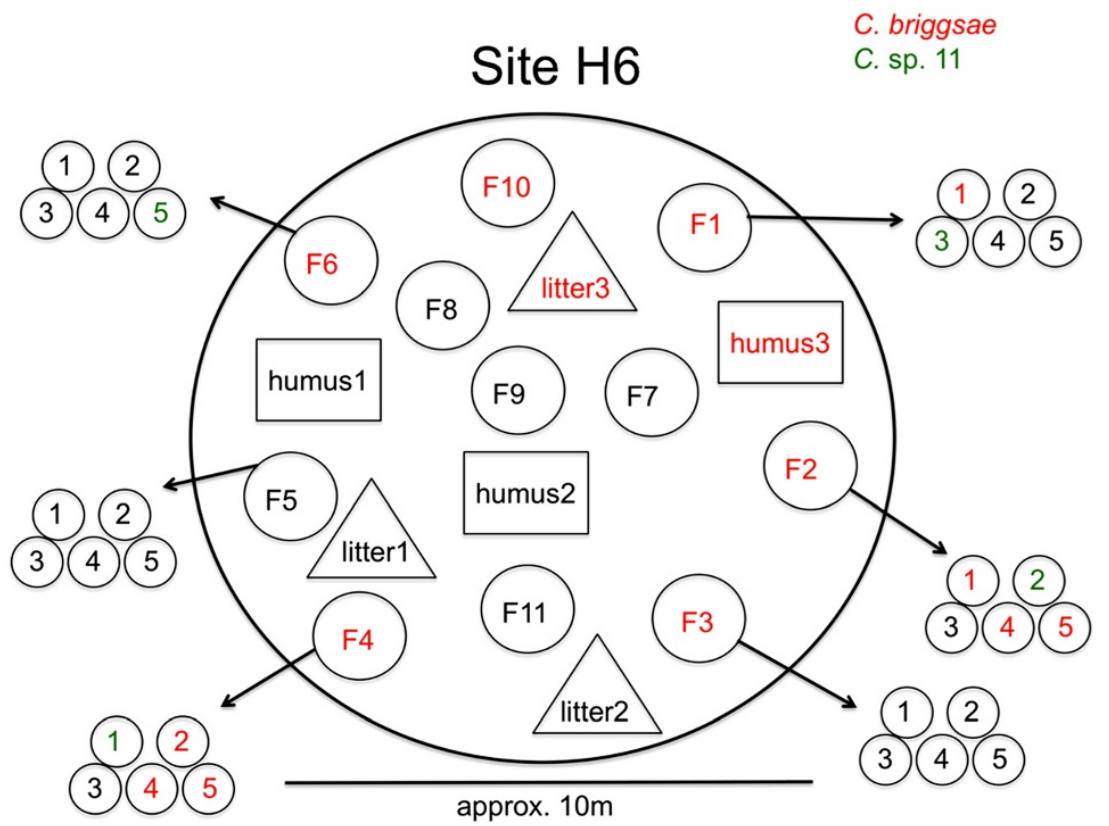

Figure 4 Small-scale spatial sampling of Cecropia fruits in Nouragues, French Guiana. Two independent sites, H3 (A) and H6 (B) were sampled. F = fruit, with subsampling of five individual "fingers", numbered 1 to 5 (see Cecropia fruit morphology in Additional file 1). In addition, leaf litter and humus were sampled at the same sites, as well as invertebrates ("inv"), such as snails.

and from other sampling sites in French Guiana. We further placed the results in the context of worldwide diversity of this species.

We first investigated the global pattern of C. briggsae diversity with a multi-locus dataset of 189 isolates, including 57 new isolates derived primarily from tropical sites (Additional files 3 and 4). Our broad-scale analysis (Figure 5A) is consistent with previous reports indicating that most $C$. briggsae isolates partition into two main groups that largely separate by latitude, the "Tropical/I" and "Temperate/II" phylogeographic groups, in addition to a few genetically distinct isolates from geographicallyrestricted localities that do not follow this latitudinal dichotomy [6,16-20]. Genetic differentiation among the phylogeographic groups is very strong in relative terms $\left(F_{S T}>0.85\right.$; Table 2$)$, although the absolute magnitude of 


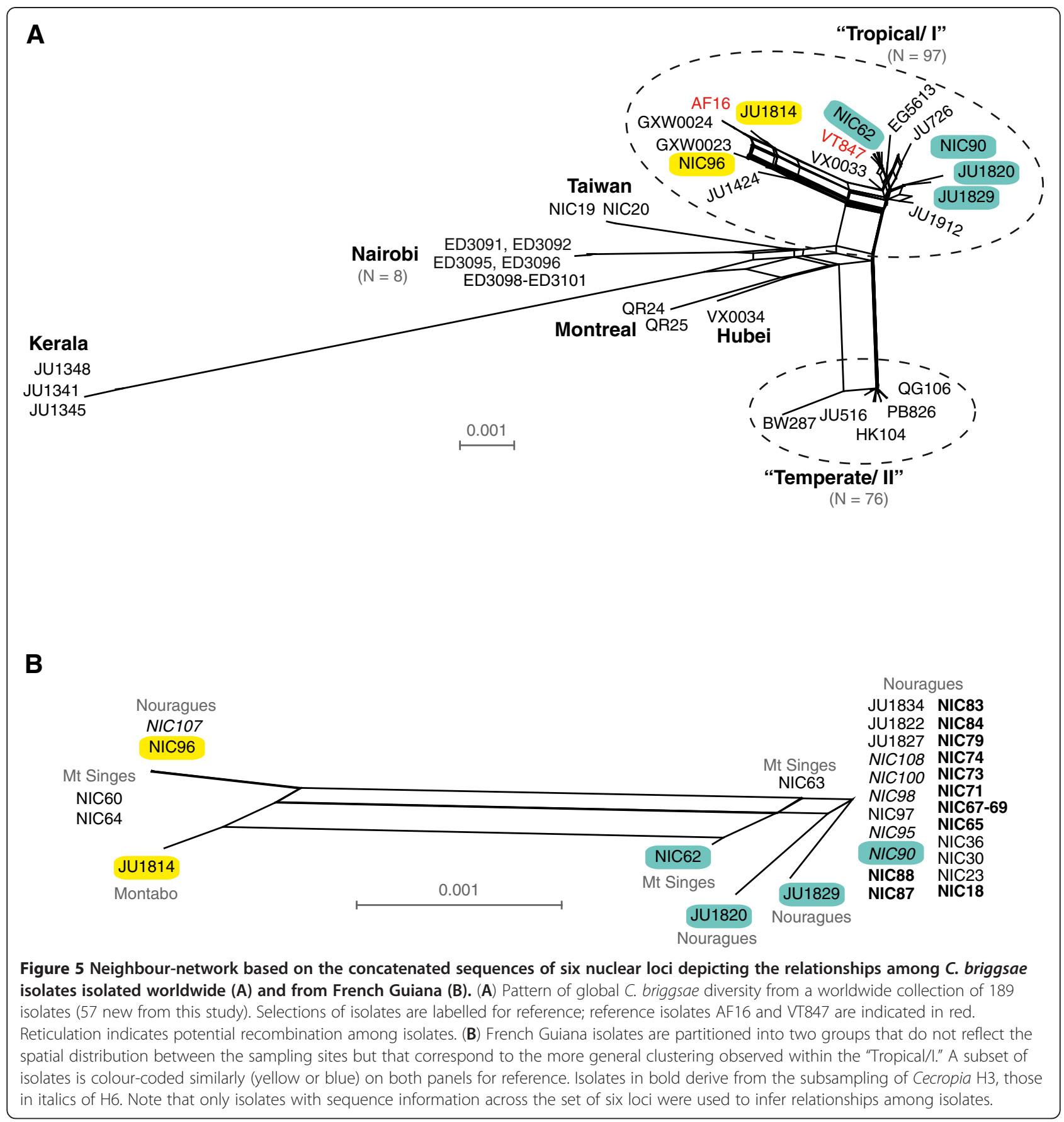

divergence between them is modest $\left(D_{x y}<1.6 \%\right)$ owing to the low species-wide polymorphism in C. briggsae. This contrasts with the weaker $F_{S T}$ and greater absolute divergence among populations in species such as $C$. remanei [21]. Here, we merge prior naming schemes for genetic groups within $C$. briggsae that were based on phylogeography with those numerical designations used for mitochondrial haplotype groups, e.g. "Tropical" as in [6] versus "clade I" as in [20], both for clarity with previous literature and to retain biologically informative labels when referring to genetic groups of C. briggsae for which there is evidence of local adaptation [22].

The genetic composition of several isolates further demonstrates that demarcation of the range boundary for the two main haplotype groups within C. briggsae does not strictly adhere to the Tropic of Cancer (Figure 5A) $[19,20]$. Specifically, Chinese isolates GXW0023 and VX0033 from Wuhan $\left(30^{\circ} \mathrm{N}\right)$ and GXW0024 from Weifang $\left(36^{\circ} \mathrm{N}\right)$ clearly fall into the "Tropical/I" haplotype group, but are found farther north than most such 
Table 2 Differentiation $\left(F_{S T}\right.$, below diagonal) and per-site divergence ( $D_{x y}$ above diagonal) between phylogeographic groups for the 6 nuclear loci combined

\begin{tabular}{lllllll}
\hline & Tropical/I & Temperate/II & Nairobi & Kerala & Taiwan & Montreal \\
\hline Tropical/I $(n=97)$ & & 0.0047 & 0.0056 & 0.0159 & 0.0059 & 0.0052 \\
Temperate/lI $(n=76)$ & 0.854 & & 0.0068 & 0.0167 & 0.0069 & 0.0063 \\
Nairobi $(n=8)$ & 0.880 & 0.989 & & 0.0150 & 0.0047 & 0.0063 \\
Kerala* $(n=3)$ & 0.936 & 0.975 & 0.972 & & 0.0159 & 0.0155 \\
Taiwan* $(n=2)$ & 0.867 & 0.974 & 0.962 & 0.967 & 0.0064 \\
Montreal $(n=2)$ & 0.877 & 0.993 & 0.994 & 0.976 & 0.978 & \\
\hline
\end{tabular}

*Only the highly differentiated strains are labelled "Kerala" and "Taiwan" (Figure 5A).

isolates, and farther north even than some isolates from the "Temperate/II" haplotype group. Despite these exceptions, $93.6 \%$ of 141 "Tropical/I" isolates were collected in tropical or subtropical latitudes, and $100 \%$ of 72 "Temperate/II" isolates were collected in temperate latitudes. Interestingly, in addition to the more common "Tropical/I" isolates, the sets from Kerala, Hubei and Taiwan each included strains corresponding to distinct haplotype groups, suggesting that sampling at local scales might reveal substantial genetic diversity $[16,19]$. This observed diversity motivated us to investigate local patterns of diversity of the C. briggsae isolates from French Guiana.

All C. briggsae isolated from French Guiana are nested within the "Tropical/I" phylogeographic group. The multilocus analysis showed that they cluster into two subgroups that mirror the pattern of differentiation observed circum-globally for "Tropical/I" isolates ("AF16-like" and "VT847-like;" Figure 5). The C. briggsae isolates from French Guiana thus harbour a great part of the genetic diversity found within "Tropical/I," despite the relatively small region sampled.

Within French Guiana, these two genetic sub-groups do not partition according to locality of origin. Indeed, diverse genotypes occurred at the two sites where several C. briggsae isolates were collected (i.e. both Nouragues and Montagne des Singes). In addition, we found little differentiation $\left(F_{S T}=0.267\right)$ and divergence $\left(D_{x y}=\right.$ 0.0020 ) between isolates from Nouragues and Montagne des Singes. Moreover, genotypes from the two genetic subgroups co-occurred at the smallest scale in the subsampling of Cecropia fruits (H6; italics in Figure 5B). Distinct genotypes also occurred at the scale of 5-10 metres in Montagne des Singes (NIC63 vs. NIC64). This result indicates that population structure among the French Guiana genotypes is not consistent with strong local population genetic differentiation that would lead to isolation by distance. Instead, the pattern of population genetic diversity of C. briggsae within French Guiana is consistent with it being a microcosm of pan-tropical diversity patterns, at least for the level of resolution permitted with single nucleotide polymorphism in nuclear loci. However, one multilocus genotype was dominant in the samples from Nouragues, which likely contributes to its non-zero differentiation with samples from Montagne des Singes (Figure 5B).

To further investigate local patterns of diversity at various spatial scales, we then compared nucleotide polymorphism of $C$. briggsae that were collected in French Guiana with isolates from several other locations. Although different genotypes were found on the same fruit in French Guiana (Figure 5B), we did not observe any nucleotide variation within a sample of isolates collected in New Jersey at a scale of a few metres (Table 3). This result is consistent with the finding that "Temperate/II" isolates harbour thirteen-fold less nucleotide polymorphism (per site $\pi_{\mathrm{si}}=0.00012$ ), on average, than isolates from "Tropical/I" $\left(\pi_{\mathrm{si}}=0.00159\right.$; Table 3) [6]. At an intermediate spatial scale, we found overall similar levels of nucleotide diversity between the C. briggsae isolates from French Guiana and isolates from the island of Maui, Hawaii (Table 3). Nevertheless, it is noteworthy that the size of the sample from Maui is six-fold smaller than that of French Guiana. Further intensive sampling of small islands is warranted to investigate total diversity in C. briggsae, which might uncover novel sequence variants. For instance, inclusion of C. briggsae isolates from several Hawaiian islands raises global measures of nucleotide polymorphism (Table 3 ).

\section{Discussion}

\section{High Caenorhabditis species richness in a tropical} rainforest

Here we documented the extensive sampling of Caenorhabditis in a tropical rainforest in French Guiana and characterized the structure of genetic diversity of the cosmopolitan species C. briggsae from small $(\sim 1 \mathrm{~m})$, intermediate $(\sim 1 \mathrm{~km})$ and up to global spatial scales. We identified six species from a single tropical site, representing nearly $25 \%$ of the 26 Caenorhabditis species known in laboratory culture [3]. Three of these species may be endemic to this region, although sampling of Caenorhabditis in South America is very limited. These findings reinforce the view that Caenorhabditis nematodes can be locally diverse and - although they have 
Table 3 Population genetic summary statistics for different sample sets of $C$. briggsae, averaged across six nuclear loci

\begin{tabular}{|c|c|c|c|c|c|c|c|}
\hline Origin* & Sample type $^{\mathrm{a}}$ & $\mathrm{n}^{\mathbf{b}}$ & $\mathrm{Hd}^{\mathrm{c}}$ & $h^{d}$ & $S^{e}$ & $\pi_{s i}(\%)^{f}$ & $\theta_{\text {si }}(\%)^{\mathrm{g}}$ \\
\hline New Jersey & Local & 13.8 & 0 & 1.0 & 0 & 0 & 0 \\
\hline Nouragues & Local & 42.7 & 0.123 & 2.8 & 3.7 & 0.045 & 0.132 \\
\hline All French Guiana & Pooled & 50.2 & 0.249 & 3.3 & 4.0 & 0.089 & 0.140 \\
\hline Maui & Local & 8.0 & 0.355 & 2.0 & 2.2 & 0.179 & 0.133 \\
\hline All Hawaii & Pooled & 12.3 & 0.452 & 2.8 & 3.8 & 0.214 & 0.203 \\
\hline Tropical/l & Pooled & 127.5 & 0.425 & 7.3 & 7.2 & 0.159 & 0.234 \\
\hline Temperate/II & Pooled & 77.7 & 0.034 & 1.8 & 1.7 & 0.012 & 0.076 \\
\hline Worldwide & Pooled & 221.5 & 0.564 & 11.3 & 20.3 & 0.403 & 0.691 \\
\hline
\end{tabular}

*See Figure 5A; " "Local" samples refer to isolates collected from a single putative population whereas "pooled" samples refer to isolates collected from disjoint populations [23,24]; ${ }^{b} \mathrm{n}$ : mean number of strains; ${ }^{\mathrm{C}} \mathrm{Hd}$ : haplotype diversity; ${ }^{\mathrm{d}} \mathrm{h}$ : number of haplotypes; ${ }^{\mathbf{e}} \mathrm{S}$ : number of segregating sites; ${ }^{\mathbf{f}} \pi_{\mathrm{si}}$ : average number of nucleotide differences per silent site; ${ }^{\mathbf{g}} \theta_{\mathrm{si}}$ : nucleotide diversity per silent site.

been generally referred to as soil nematodes - are more easily found in rotting vegetal matter, especially fruits, flowers and stems $[3,13]$.

Our sampling can be contrasted with a recent extensive sampling effort in temperate France [13]. In mainland France, just two species of Caenorhabditis are found commonly: C. elegans and C. briggsae. In addition, $C$. remanei and $C$. sp. 13 each have been isolated once in the course of sampling many locations in France over many years [13]. The isolation of six distinct Caenorhabditis species in just a few square kilometres of tropical forest, including three potentially endemic species, testifies to much higher species richness of Caenorhabditis in the tropics [3]. Moreover, this finding for Caenorhabditis is fully consistent with latitudinal diversity patterns documented for many other taxa $[25,26]$. As discovery of new species in this group is progressing rapidly, phylogenetically-informed analysis of Caenorhabditis species distributions may shed light on long-standing questions about the causes of latitudinal species diversity gradients [27].

Previous sporadic sampling of Caenorhabditis nematodes in Tropical South and Central America has yielded C. sp. 11, C. brenneri, and C. briggsae. An additional three species have also been found on Caribbean islands (C. sp. 14, C. sp. 19, and C. sp. 20) [3]. The absence of the well-known C. elegans in the Nouragues Natural Reserve is not surprising, however, given that this species is rare in previous sampling from the tropics [3]. Thus, the distribution of Caenorhabditis species around the world is consistent with there being a few cosmopolitan species and many other species endemic to particular geographic regions.

Our study demonstrates the first evidence of heterogeneous species distributions of Caenorhabditis at an intermediate spatial scale on the order of kilometres. It will be important in future work to determine whether these species distributions reflect stable features that might correspond to distinct ecological habitat characteristics of the different species, or instead reflect stochastic local abundances that are dynamic in time, perhaps owing to strong metapopulation dynamics associated with ephemeral food patches that can be utilized by many Caenorhabditis species. Co-occurrence of multiple species was common for samples of the same substrate type, and sometimes within the same sample, a pattern also reported for the less species-rich sampling in mainland France [13]. These observations raise the neglected issue of niche space in Caenorhabditis biology as a major question to be answered to understand the abundance and distribution of species. Unfortunately, there are few data describing Caenorhabditis fitness reaction norms in relation to potential ecological factors that could differentially limit species persistence (e.g. temperature, bacterial food specificity, substrate chemistry) [13,22,28-30].

\section{Spatial structure of genetic diversity}

We used the cosmopolitan C. briggsae as a focal taxon for quantifying population genetic variability from the finest sampling scales within the Nouragues forest samples up to pooled samples at regional and global scales. We found that distinct $C$. briggsae genotypes co-exist at scales down to a few metres (Montagne des Singes) or even a single fruit (Nouragues, sample H6). Given their highly selfing mode of reproduction in nature $[6,13]$, this suggests that a given fruit can be colonized by multiple individuals of distinct genetic backgrounds. These results mirror findings for temperate latitude samples of C. elegans, in that high local diversity may occur while genetic differentiation among locations is low $[8,10,11,14,31,32]$. This pattern of large-scale homogeneity coupled with fine-scale heterogeneity also is reminiscent of the 'chaotic genetic patchiness' observed for some intertidal invertebrates with a highly dispersing planktonic stage [33]. In contrast to $C$. elegans, however, strong population structure dominates the global scale in C. briggsae, with 1) a large "Tropical/ I" phylogeographic group distributed widely in the tropics around the world, 2) a large "Temperate/II" 
phylogeographic group having very low polymorphism overall and hence undetectable local diversity, and 3) a few genetically divergent clades, so far each found only in one place (Nairobi, Kenya; Montreal, Canada; Tai-an, Taiwan; Hubei, China; Kerala, India) [16,19]. It is likely that such geographically restricted diversity is not yet exhausted by our sampling, nor are the possibilities for observing recombination among the main phylogeographic groups. Recombination had been previously noted at this large scale $[6,20]$ and can also be inferred among the French Guiana multilocus genotypes, as visualized by the reticulation pattern of the haplotype network (Figure 5B).

\section{Conclusions}

In this study, we described the first sampling of Caenorhabditis in a primary tropical forest environment, analysing a variety of substrates. We found six Caenorhabditis species, mostly in rotting flowers and fruits, the densest richness of species known in this group. Three of these species are cosmopolitan, three appear less widespread and might be endemic to French Guiana. The genetic structure of $C$. briggsae populations indicates events of long-distance migrations among different continents in the tropical zone, and a local spatial structure resembling that described for C. elegans in temperate zone populations.

\section{Methods}

\section{Sampling sites in French Guiana}

Samples were collected between November 17 and 27, 2009 at the end of the dry season. Most samples were collected at the CNRS "Inselberg" field station in the Nouragues Natural Reserve $\left(4.08809^{\circ} \mathrm{N},-52.6796^{\circ} \mathrm{W}\right)$ within an area of approximately four square kilometres (Figure 3). We also collected samples at other locations, including mangrove forests in the outskirts of Cayenne (Montabo trail), secondary forests (e.g. Montagne des Singes, Kourou), and agricultural areas around the village of Cacao (Figure 1).

\section{Sample collection and analysis}

We collected a variety of rotting plant material, primarily targeting rotting fruits and flowers. Such substrates are known to frequently harbour Caenorhabditis [3] (Additional files 1 and 2). Samples were collected, photographed and stored in plastic zip bags. A given sample identifier code refers to a substrate sample (e.g. a single or multiple fruits) at a single location of a few square metres, contained within a single bag. Part of the samples were analysed at the Nouragues field station using a dissecting microscope and ready-to-use $35 \mathrm{~mm}$ C. elegans Normal Growth Medium (NGM) culture plates previously seeded with E. coli OP50 [34], allowing us to determine developmental stages and number of individuals present at the time of isolation. The other samples were stored for the duration of the field trip (14 days) and examined afterwards in the laboratories in Nice and Paris, using 55 and $90 \mathrm{~mm}$ culture plates. Samples were spread around the lawn and 1-2 $\mathrm{ml}$ of water or M9 solution was added to the samples. All plates were examined regularly using the dissecting microscope, and nematodes were isolated over a period of seven days. Note that all C. briggsae isolates used for genetic analysis (see below) were derived from independent substrate samples, except for one sample (G2) from which eight $C$. briggsae isolates were derived from different individuals, immediately after isolation on site (isolates NIC23, NIC28, NIC29, NIC30, NIC36, NIC37, NIC38, NIC40) (Additional file 2). This sampling protocol ensured that any potential genetic differences found among isolates indicate the presence of genetically distinct natural isolates in the same substrate sample.

\section{Species identification and isolate establishment}

Caenorhabditis genus identity was confirmed by microscopy using morphological criteria [34]. Species identity was further examined through mating tests with known species and/or ITS2 sequencing as described previously [3]. (Note that newly discovered species are attributed a number before a formal taxonomic description has been published). Stock cultures were established by isolation of a single hermaphrodite or female. For gonochoristic species, we picked either a female with a copulatory plug, or one female and one male. The mode of reproduction was determined by isolating virgin L4 females/hermaphrodites and scoring for the absence/presence of progeny. For androdioecious species, isogenic strains (referred to as "isolate") were produced by isolating a single larva for a few (3-6) generations. For gonochoristic species, strains were established as isofemale lines. From each isolated Caenorhabditis sample, we established cultures, which were cryopreserved and stocked in our collections.

A complete list of isolates can be found in Additional file 2. JU isolates are stored in the lab of MAF and NIC isolates are stored in the lab of CB. All worm strains are available upon request.

\section{Geographically localised ("small-scale") sampling of Cecropia fruits in the Nouragues forest}

To examine the species distribution and genetic diversity of C. briggsae at different spatial scales, we collected a large number of samples from rotting fruits of Cecropia sp. (likely Cecropia sciadophylla) at two sites in the Nouragues Natural Reserve from which we had previously collected C. briggsae and other Caenorhabditis species (Sample ID H3, H6; Additional file 2). The forest ground at these two sites was covered by numerous rotting Cecropia fruits of black or sometimes brown colour. 
At each site, we collected 11 individual rotting fruits from the ground within an area of approximately $40 \mathrm{~m}^{2}$ (fruits were separated from each other by approx. 2-8 m). From six of these fruits, we derived sub-samples by collecting separately five small fruit parts ("fingers") from each fruit. From both sampling sites, we also collected three litter samples (consisting primarily of rotting leaves) and three humus samples. For one of the sites (samples H3), we further collected four invertebrate samples: one live snail, two snail shells, and one live myriapod. Collection of independent subsamples of each substrate sample on site allowed subsequent analysis to detect potential mixing of different genotypes in the same sample. For details of sampling design, see Figure 4.

\section{Additional C. briggsae isolates analysed}

We further included 46 new C. briggsae isolates, mostly collected from rotting vegetation, from localities across five continents; including eight regions not previously sampled for Caenorhabditis nematodes (Additional file 3). Each isolate was founded from a single hermaphrodite individual, maintained using standard protocols, and taxonomic status was determined by mating tests and/or ITS2 sequencing [3]. The geographic and ecological information of the wild isolates was also entered in Wormbase (http://www.wormbase.org). An interactive map of collection localities and isolate information can be found online (C. briggsae: https://www.google.com/maps/ $\mathrm{ms} ? \mathrm{msa}=0 \& \mathrm{msid}=117700919974655793194.00046 \mathrm{c} 7 \mathrm{ccc} 0 \mathrm{a}$ fcc $319 b 7 \& t=h \& z=2)$.

\section{DNA amplification and sequencing}

Gene fragments corresponding to the six loci used in previous studies of $C$. briggsae diversity were amplified and sequenced using published primers [6]. DNA was isolated from large populations of isogenic worms of each strain using the DNeasy Blood and Tissue kit (Qiagen). Amplifications were processed in $30 \mu \mathrm{L}$ reaction volumes with $1.5 \mu \mathrm{L}$ DMSO, $3 \mu \mathrm{L}$ dNTPs $(6.6 \mathrm{mM})$, $3 \mu \mathrm{L}$ 10X Buffer (Fermentas), 2.4 $\mu \mathrm{L} \mathrm{MgCl}_{2}, 0.36 \mu \mathrm{L}$ of each primer $(50 \mu \mathrm{M}), 0.18 \mu \mathrm{L}$ of Taq polymerase (New England Biolabs) and $2 \mu \mathrm{L}$ of genomic DNA. Cycling conditions were: $95^{\circ} \mathrm{C}$ for 4 min followed by 35 cycles of $95^{\circ} \mathrm{C}$ for $1 \mathrm{~min}, 55^{\circ} \mathrm{C}$ or $58^{\circ} \mathrm{C}$ for $1 \mathrm{~min}$ and $72^{\circ} \mathrm{C}$ for 1 min. Amplifications were sequenced at the University of Arizona UAGC sequencing facility. All markers were sequenced on both strands and all polymorphisms were visually verified using sequencing chromatograms. Primer sequences were manually deleted from each sequence prior to analysis. All new sequences are accessible in GenBank with accession numbers JX288123JX288761.

\section{Sequence analyses}

We analysed a total of 99 new wild isolates of C. briggsae (53 from French Guiana, 46 from other world-wide localities). Note that four French Guiana isolates (NIC88, NIC106, NIC107, NIC108) were analysed in Jovelin \& Cutter [19]. Based on a set of six nuclear loci used previously $[6,16,17,19]$, we investigated the relationships of the 57 new isolates for which data were collected for all six loci, relative to a collection of 132 isolates that were used in previous studies on C. briggsae diversity $[6,16,17,19]$. We also obtained sequence data for up to five of these loci for the remaining new isolates, which informed some analyses (Additional file 3). Multiple sequence alignments were constructed for each locus in BioEdit [35] and manually edited for indel polymorphisms. We inferred the relationships among isolates with concatenated sequences from the set of six loci using unrooted neighbournetworks generated with a Jukes-Cantor distance in the program SplitsTree 4.10 [36]. Neighbour-networks are useful for representing the relationships among individuals of the same species for which recombination events may be non-negligible [37]. We then measured differentiation and divergence between phylogeographic groups using $F_{S T}$ [38] and $D_{x y}$, the average number of nucleotide substitutions per site between groups [39]. We also measured nucleotide polymorphism [39] at each locus taken separately using DnaSP v.5 [40] and took the average across loci in order to investigate nucleotide diversity at multiple spatial scales in C. briggsae.

\section{Additional files}

Additional file 1: Photographs of substrate samples from French Guiana containing Caenorhabditis. Description: From left to right and top to bottom: Heliconia flower, rotting Cecropia fruits, Ficus sp. (sample A1), rotten Eperua falcata flowers (sample D34), Clusia grandiflora fruits, rotten flowers (sample D14), Inga sp. fruit (F9), yellow fungus (sample D10). Most of the photographed fruits and flowers were less decayed than those sampled (with the exception of Heliconia, where the rotting matter inside erect flowers was collected).

Additional file 2: List of samples from French Guiana along with corresponding isolate and species analysis (three sheets).

Description: The first sheet "Nouragues" lists all samples collected at the Nouragues field station. These samples were mostly analysed on site within a few hours after isolation, allowing for Caenorhabditis population census and developmental stage assessment. The column "GPS ID" correspond to GPS reference numbers reported on the map in Figure 3. The column "Date sampling" indicates the day when the substrate sample was collected and the column "Sample analysis" indicates the time point at which samples were placed on culture plates for analysis. The column "Caenorhabditis population 12 hrs after" describes population census and developmental stages observed (only for samples that were examined on site, immediately after isolation); L1-L4: first to fourth larval stage, ad: adult. Species and isolate derived from a given sample are listed in the last column "Species and Strains". The second sheet "Cecropia subsampling" lists all samples collected for the detailed geographically localised ("small-scale") sampling of Caenorhabditis nematodes in rotting Cecropia fruits at two localities ( $\mathrm{H3}$ and $\mathrm{H} 6)$ at the Nouragues Field station. For details on sampling scheme, see Figure 4 and the Materials and Methods section. The third sheet "French Guiana" 
details all samples collected outside the Nouragues area in other parts of French Guiana. None of these samples were analysed on site. In all sheets, positive samples, i.e. samples containing Caenorhabditis, are highlighted with an orange background.

\section{Additional file 3: List of new C. briggsae isolates used to investigate} nucleotide diversity. Description: The first sheet "C. briggsae isolates" lists all new C. briggsae isolates used to examine nucleotide diversity ( $N=99 ; 53$ from French Guiana, 46 from other world-wide localities). Note that four French Guiana isolates (NIC88, NIC106, NIC107, NIC108) were already analysed in Jovelin \& Cutter [19] and are therefore not listed. For detailed sampling information on the previously examined C. briggsae isolates $(N=132)$, see $[6,16,17,19]$. The last column indicates the list of sequenced loci for each isolate. Only isolates with sequence information across the set of six loci were used to infer relationships among isolates, while all isolates were used to investigate patterns of nucleotide diversity. The coloured background indicates all isolates from a same subsampling in Nouragues: $\mathrm{H} 3$ subsampling (yellow), $\mathrm{H} 6$ subsampling (green), and isolates derived from a single substrate sample, G2 (blue). The second sheet "Primers" indicates primer information and corresponding genomic regions from [6,38]

Additional file 4: Haplotype structure of $C$. briggsae isolates from a worldwide distribution based on six nuclear loci. Description: Only the polymorphic sites and indels are included. All indels are shown by a dash, regardless of length. Dots represent nucleotides identical to those shown in the top sequence. Gene names are indicated in the bottom panel with linkage groups (LG) indicated in the top panel. The number of isolates along with the isolate name is shown on the right for each haplotype. Newly sequenced isolates are labelled in bold.

\section{Competing interests}

The authors declare that they have no competing interests.

\section{Authors' contributions}

MAF and CB collected the French Guiana samples, and analysed them with the help of CF. ECA collected and analysed the Maui samples. RJ, SH and YRC collected sequence data and RJ analysed them. $C B, M A F, R J$ and $A D C$ wrote the manuscript. CB, MAF, ADC designed and supervised the research. All authors read and approved the final manuscript.

\section{Acknowledgements}

MAF and CB acknowledge financial support by the CNRS, including a grant for research at the Nouragues field station, and would like to thank the staff at the station and the CNRS in French Guiana, in particular, Patrick Châtelet, Philippe Gaucher and Jérôme Chave. We also thank those who provided access to sampling sites (Bruno Le Vessier) and information on sampled fruits (Pierre-Michel Forget). We are grateful to Matt Rockman for the New Jersey isolates and to Patrick Châtelet, Mathieu Joron, Didier Peigne, Christopher Nelson, Amir Yassin for collecting samples. CB is funded by the CNRS, Agence Nationale de la Recherche and the Fondation Schlumberger pour I'Education et la Recherche. ADC received support from the Natural Sciences and Engineering Research Council (NSERC) of Canada and from a Canada Research Chair. RJ was supported by an Ontario Ministry of Research and Innovation postdoctoral fellowship and SH was supported by a University of Toronto Excellence Award. ECA was supported by a Ruth L. Kirschstein National Research Service Award post-doctoral fellowship (F32-GM089007) and training grant T32-CA009528 from the National Cancer Institute.

\section{Author details}

${ }^{1}$ Institut de Biologie de l'Ecole Normale Supérieure, CNRS - ENS - INSERM, 46 rue d'Ulm, Paris cedex 05 75230, France. 'Department of Ecology and Evolutionary Biology, University of Toronto, 25 Willcocks St, Toronto, ON M5S 3B2, Canada. Institut de Biologie Valrose, CNRS, UMR7277, Parc Valrose, Nice cedex 02 06108, France. ${ }^{4}$ INSERM, U1091, Nice cedex 02 06108, France. ${ }^{5}$ University of Nice Sophia Antipolis, UFR Sciences, Nice cedex 0206108 France. ${ }^{6}$ Department of Ecology and Evolutionary Biology, Lewis-Sigler Institute for Integrative Genomics, Princeton University, Princeton, NJ, USA.

Received: 9 October 2012 Accepted: 7 January 2013

Published: 12 January 2013

\section{References}

1. Cutter AD, Dey A, Murray RL: Evolution of the Caenorhabditis elegans genome. Mol Biol Evol 2009, 26(6):1199-1234.

2. Félix M-A, Braendle C: The natural history of Caenorhabditis elegans. Curr Biol 2010, 20:R965-R969.

3. Kiontke K, Félix M-A, Ailion M, Rockman MV, Braendle C, Pénigault J-B, Fitch $\mathrm{DH}$ : A phylogeny and molecular barcodes for Caenorhabditis, with numerous new species from rotting fruits. BMC Evol Biol 2011, 11:339.

4. Kiontke K, Sudhaus W: Ecology of Caenorhabditis species. In Wormbook: The C. elegans Research Community; 2006. http://www.wormbook.org.

5. Barrière A, Félix M-A: Natural variation and population genetics of $C$. elegans. In Wormbook: The C. elegans Research Community; 2005. http:// www.wormbook.org.

6. Cutter AD: Nucleotide polymorphism and linkage disequilibrium in wild populations of the partial selfer Caenorhabditis elegans. Genetics 2006, 172(1):171-184.

7. Graustein A, Gaspar JM, Walters JR, Palopoli MF: Levels of DNA polymorphism vary with mating system in the nematode genus Caenorhabditis. Genetics 2002, 161(1):99-107.

8. Haber M, Schüngel M, Putz A, Müller S, Hasert B, Schulenburg H: Evolutionary history of Caenorhabditis elegans inferred from microsatellites: evidence for spatial and temporal genetic differentiation and the occurrence of outbreeding. Mol Biol Evol 2005, 22:160-173.

9. Jovelin R, Ajie BC, Phillips PC: Molecular evolution and quantitative variation for chemosensory behaviour in the nematode genus Caenorhabditis. Mol Ecol 2003, 12(5):1325-1337.

10. Sivasundar A, Hey J: Sampling from natural populations with RNAI reveals high outcrossing and population structure in Caenorhabditis elegans. Curr Biol 2005, 15(17):1598-1602.

11. Barrière A, Felix M-A: Temporal dynamics and linkage disequilibrium in natural C. elegans populations. Genetics 2007, 176:999-1011.

12. Caswell-Chen EP, Chen J, Lewis EE, Douhan GW, Nadler SA, Carey JR: Revising the standard wisdom of $C$. elegans natural history: ecology of longevity. Sci Aging Knowl Environ 2005, 40:pe30.

13. Félix MA, Duveau F: Population dynamics and habitat sharing of natural populations of Caenorhabditis elegans and C. briggsae. BMC Biol 2012, 10(1):59.

14. Andersen EC, Gerke JP, Shapiro JA, Crissman JR, Ghosh R, Bloom JS, Felix MA, Kruglyak L: Chromosome-scale selective sweeps shape Caenorhabditis elegans genomic diversity. Nat Genet 2012, 44:285-290.

15. Phillips PC: Self-fertilization sweeps up variation in the worm genome. Nat Genet 2012, 44(3):237-238.

16. Cutter AD, Yan W, Tsvetkov N, Sunil S, Felix MA: Molecular population genetics and phenotypic sensitivity to ethanol for a globally diverse sample of the nematode Caenorhabditis briggsae. Mol Ecol 2010, 19(4):798-809.

17. Dolgin ES, Felix MA, Cutter AD: Cutter Hakuna Nematoda: genetic and phenotypic diversity in African isolates of Caenorhabditis elegans and C. briggsae. Heredity 2008, 100(3):304-315.

18. Howe DK, Denver DR: Muller's Ratchet and compensatory mutation in Caenorhabditis briggsae mitochondrial genome evolution. BMC Evol Biol 2008, 8:62.

19. Jovelin R, Cutter AD: MicroRNA sequence variation potentially contributes to within-species functional divergence in the nematode Caenorhabditis briggsae. Genetics 2011, 189(3):967-976.

20. Raboin MJ, Timko AF, Howe DK, Felix MA, Denver DR: Evolution of Caenorhabditis mitochondrial genome pseudogenes and Caenorhabditis briggsae natural isolates. Mol Biol Evol 2010, 27(5):1087-1096.

21. Dey A, Jeon Y, Wang GX, Cutter AD: Global population genetic structure of Caenorhabditis remanei reveals incipient speciation. Genetics 2012, 191(4):1257-1269.

22. Prasad A, Croydon-Sugarman MJ, Murray RL, Cutter AD: Temperaturedependent fecundity associates with latitude in Caenorhabditis briggsae. Evolution 2011, 65(1):52-63.

23. Stadler T, Haubold B, Merino C, Stephan W, Pfaffelhuber P: The impact of sampling schemes on the site frequency spectrum in nonequilibrium subdivided populations. Genetics 2009, 182(1):205-216.

24. Cutter AD, Wang GX, Ai H, Peng Y: Influence of finite-sites mutation, population subdivision and sampling schemes on patterns of nucleotide polymorphism for species with molecular hyperdiversity. Mol Ecol 2012, 21(6):1345-1359. 
25. Gaston KJ: Global patterns in biodiversity. Nature 2000, 405(6783):220-227.

26. Mittelbach GG, Schemske DW, Cornell HV, Allen AP, Brown JM, Bush MB, Harrison SP, Hurlbert AH, Knowlton N, Lessios HA, et al: Evolution and the latitudinal diversity gradient: speciation, extinction and biogeography. Ecol Lett 2007, 10(4):315-331.

27. Weir JT, Schluter D: The latitudinal gradient in recent speciation and extinction rates of birds and mammals. Science 2007, 315(5818):1574-1576.

28. Anderson JL, Albergotti L, Ellebracht B, Huey RB, Phillips PC: Does thermoregulatory behavior maximize reproductive fitness of natural isolates of Caenorhabditis elegans? BMC Evol Biol 2011, 11:157.

29. Fodor A, Riddle DL, Nelson FK, Golden JW: Comparison of a new wild-type Caenorhabditis briggsae with laboratory strains of Caenorhabditis briggsae and Caenorhabditis elegans. Nematologica 1983, 29:203-217.

30. Grewal PS: Influence of bacteria and temperature on the reproduction of Caenorhabditis elegans (Nematoda: Rhabditidae) infesting mushrooms (Agaricus bisporus). Nematologica 1991, 37:72-82.

31. Barrière A, Félix M-A: High local genetic diversity and low outcrossing rate in Caenorhabditis elegans natural populations. Curr Biol 2005 . 15:1176-1184

32. Cutter $A D$, Félix M-A, Barrière $A$, Charlesworth D: Patterns of nucleotide polymorphism distinguish temperate and tropical wild isolates of Caenorhabditis briggsae. Genetics 2006, 173:2021-2031.

33. Johnson MS, Black R: Chaotic genetic patchiness in an intertidal limpet, Siphonaria sp. Mar Biol 1982, 70:157-164.

34. Barrière A, Félix M-A: Isolation of $C$. elegans and related nematodes. In Wormbook: The C. elegans Research Community; 2006. http://www. wormbook.org].

35. Hall TA: BioEdit: a user-friendly biological sequence alignment editor and analysis program for Windows 95/98/NT. Nucleic Acids Symp Ser 1999, 41:95-98.

36. Huson DH, Bryant D: Application of phylogenetic networks in evolutionary studies. Mol Biol Evol 2006, 23(2):254-267.

37. Huson DH, Scornavacca C: A survey of combinatorial methods for phylogenetic networks. Genome Biol Evol 2011, 3:23-35.

38. Wright S: The genetical structure of populations. Ann Eugenics 1951, 15:323-354

39. Nei M: Molecular Evolutionary Genetics. New York: Columbia University Press; 1987.

40. Librado P, Rozas J: DnaSP v5: a software for comprehensive analysis of DNA polymorphism data. Bioinformatics 2009, 25(11):1451-1452.

doi:10.1186/1471-2148-13-10

Cite this article as: Félix et al:: Species richness, distribution and genetic diversity of Caenorhabditis nematodes in a remote tropical rainforest. BMC Evolutionary Biology 2013 13:10.

\section{Submit your next manuscript to BioMed Central and take full advantage of:}

- Convenient online submission

- Thorough peer review

- No space constraints or color figure charges

- Immediate publication on acceptance

- Inclusion in PubMed, CAS, Scopus and Google Scholar

- Research which is freely available for redistribution 